\title{
Extension of non-equilibrium modeling of metal ablation to the thermal effects inside the target material using a two temperature model
}

\author{
Amina Ait oumeziane ${ }^{(1)}$ and Jean-Denis Parisse ${ }^{(2),(3)}$ \\ (1) Centre RAPSODEE UMR CNRS 5302, IMT mines Albi-Carmaux, France. \\ (2) IUSTI UMR CNRS 7343,Aix-Marseille university, Marseille, 13013, France \\ (3) French Air Force Academy, Salon de Provence,13300, France
}

\begin{abstract}
The aim of this paper is to contribute to the understanding of short laser ablation of metals by means of a 1D two temperature model(TTM) coupled to a 1D hydrodynamical out of equilibrium one. Thermal equilibrium inside the material is a widely admitted hypothesis in ns metal ablation. Reconsidering it, has been shown to have serval non negligeable impacts on the entire ablation process. Instead of using the Saha-Boltzman equation and the hypothesis of thermal equilibrium for the evaluation of the electrons density in the ablated matter, the TTM allows to evaluate it directly. This improves the adequacy of the simulation. Indeed, the calculated plasma ignition threshold is closer to experimental data. The present study extends previous investigations on pure metals ablation by UV short laser pulses, its purpose is to understand how the entire ablation process is affected considering the presence of thermal non-equilibrium inside the target material. Ablation depths along with thermal non-equilibrium rate variation inside the material are examined. Plasma ignition threshold and characteristics are studied as a function of the out of equilibrium rate between the electrons and the lattice of the material. Copper $(\mathrm{Cu})$ is the considered target for the development of this comprehensive modeling approach meant to describe pulsed laser ablation under several usual conditions, which remains the principal motivation of the current investigation.
\end{abstract}

\section{Nomenclature}

$T_{a} \quad$ Lattice temperature

$G \quad$ Free electrons and lattice coupling coefficient

$T_{e} \quad$ Electronic Temperature

$C_{e} \quad$ Electronic specific heat

$\kappa_{e} \quad$ Electronic thermal conductivity

$C_{a} \quad$ Lattice specific heat

$T_{h} \quad$ Heavy particles temperature

$x \quad$ Position in the plume

$t$ time

$\rho_{i} \quad$ density of the $\mathrm{i}^{\text {th }}$ species

$\rho \quad$ total density

$u \quad$ plume velocity

$P \quad$ mixture pressure

$P_{e} \quad$ Electronic pressure

$P_{h} \quad$ Heavy particles pressure

$E \quad$ Heavy particles energy

$E_{e} \quad$ Electronic energy

$k$ Boltzmann's constant

$\mathcal{N}$ Avogadro's number 
$I_{H} \quad$ The hydrogen ionization potential

$\mathrm{E}^{+} \quad$ Ionization energy

$\mathcal{M}$ Molar mass

\section{Introduction}

Copper is an abundant material which, due to its properties namely thermal and electrical conductivity is widely used especially in electronical devices and aerospace engins. After their end of life satellites disintegrat leaving behind an enourmous amount of space debris. Those debris consist mainly of metals such as copper.

Several research has been dedicated to the study of the feasiblity of space debris removal using pulsed laser beams ${ }^{1-4}$ and have all shown that the understanding of the processes governing the laser-target interaction as well as plasma formation and expansion is crucial for an effective debris removal.

In this paper UV nanosecond laser ablation of copper is investigated with a new upgraded model, consisting of a two temperature model coupled to a an out of equilibrium hydrodynamical one. Comparison with old studies using the same modeling approach under the hypothesis of thermal equilibrium inside the material ${ }^{7-11}$ are presented.

In those previous investigations plume initialisation was made under the hypothesis of local thermal equilibrium. Hence electrons initial density was evaluated using the well known Saha equation.

In this work plume initialisation was handled differently. Electrons initial density was calculated based ... detailed will be presented in the section dedicated to the model.

This model is inspired by Parisse et $\mathrm{al}^{5}$ thermal/hydrodynamical out of equilibrium model applied to short laser ablation of silicon along with Shäfer et $\mathrm{al}^{6}$ considered thermal out of equilibrium and a ther$\mathrm{mal} / \mathrm{molecular}$ dynamic simulation of ultrashort laser ablation of copper.

\section{Model}

\section{A. laser-target interaction}

Depending on the interaction conditions (the laser pulse duration (short/utlra-short, the target material nature (metal, semiconductor)) thermal effects inside the material might occur out of an equilibrium state. In that case and in order to rigorously describe the thermal effects induced by the pulsed laser beam inside the target material a two temperature model can be used ${ }^{6}$

$$
\begin{gathered}
\frac{\partial H}{\partial t}=G\left(T_{e}-T_{a}\right) \\
C_{e} \frac{\partial T_{e}}{\partial t}=\frac{\partial\left(\kappa_{e} \frac{\partial T_{e}}{\partial x}\right)}{\partial x}-G\left(T_{e}-T_{a}\right)+S_{q}
\end{gathered}
$$

where $H=C_{a} T_{a}$ is the lattice enthalpy with $C_{a}$ and $T_{a}$ being the lattice specific heat and temperature respectively. $G$ is the free electrons and lattice coupling coefficient, $C_{e}$ and $T_{e}$ the electrons specific heat and the electronic temperature, $T_{a}$ the lattice temperature and $\kappa_{e}$ the electrons thermal conductivity.

$$
\begin{aligned}
& C_{a}=3.510^{6} \mathrm{~J} / \mathrm{m}^{3} \mathrm{~K} \\
& C_{e}=\gamma T_{e} J / m^{3} K \\
& \gamma=96.6 \mathrm{~J} / \mathrm{m}^{3} \mathrm{~K}^{2} \text { and } G=10^{17} \mathrm{~W} / \mathrm{m}^{3} \mathrm{~K} \\
& S_{q}=\alpha_{0} I(x, t), \\
& I(x, t)=(1-\Re) I_{0}(t) e^{-\alpha_{0} x},
\end{aligned}
$$

where $\Re$ is the reflectivity of the irradiated surface, and $I_{0}(t)$ is the incident laser intensity. The later is modeled by an ideal Gaussian temporal profile, ${ }^{7-11} \alpha_{0}$ is the material absorption coefficient which depends on the laser beam wavelength. 


$$
\kappa_{e}=377 \cdot \frac{\left(\theta_{e}^{2}+0.16\right)^{1.25}\left(\theta_{e}^{2}+0.44\right) \theta_{e}}{\left(\theta_{e}^{2}+0.092\right)^{0.5}\left(\theta_{e}^{2}+0.139 \theta_{a}\right)}
$$

where $\theta_{e}=\frac{T_{e}}{T_{F}}$ and $\theta_{a}=\frac{T_{a}}{T_{F}}$ with $T_{F}=8.12 \cdot 10^{14} \mathrm{~K}$ is copper Fermi temperature.

\section{B. plume formation and expansion}

The model we are about to present is applied to the study of laser ablation of a copper target with a 193 nm-8 ns laser pulse in a helium background gas.

The following processes were considered:

- excitation by electronic impact

$\mathrm{Cu}+e^{-}+3.79 \mathrm{eV} \leftrightarrow \mathrm{Cu}^{*}+e^{-}$,

- ionization of the excited state by electronic impact

$\mathrm{Cu}^{*}+e^{-}+3.93 \mathrm{eV} \leftrightarrow \mathrm{Cu}^{+}+e^{-}+e^{-}$,

- photoionization of the excited state

$\mathrm{Cu}^{*}+\mathrm{h} \nu \rightarrow \mathrm{Cu}^{+}+e^{-}$,

- ionization of the singly ionized state by electronic impact

$\mathrm{Cu}^{+}+e^{-}+20.29 \mathrm{eV} \leftrightarrow \mathrm{Cu}^{+2}+e^{-}+e^{-}$,

The intermediate excited state was chosen to enable the photoionization process and to initiate plume breakdown.

The adequacy of the previously listed reactions has been proven in several works, ${ }^{5,14,16}$ from which our approach is inspired. The good agreement with experimental data of our predicted plasma ignition threshold in our previous works ${ }^{8,9}$ strongly confirms their relevance.

The kinetic constants corresponding to each one of the above mentioned processes have been calculated according to the principle of detailed balance ${ }^{15,16}$ and are summarized as follows:

The subscripts 1 to 6 are used to identify the different species present in the plume $(1: \mathrm{Cu}, 2: \mathrm{He}, 3$ : $\left.\mathrm{Cu}^{*}, 4: \mathrm{Cu}^{+}, 5: \mathrm{Cu}^{+2}, 6: \mathrm{e}^{-}\right)$

The electronic impact excitation kinetic constants in the forward and backward cases, are respectively:

$$
\begin{gathered}
K_{f-e}=6 \times 10^{-12} \times T_{e}^{0.5}\left(2+\frac{E^{*}}{k T_{e}}\right) \frac{\rho_{1} \rho_{6} \times \mathcal{N}^{2}}{\mathcal{M}_{1} \mathcal{M}_{6}} \exp \left(-\frac{E^{*}}{k T_{e}}\right)\left(\mathrm{gcm}^{-3} \mathrm{~s}^{-1}\right) \\
K_{e-f}=1.8 \times 10^{-11} \times T_{e}^{0.5}\left(2+\frac{E^{*}}{k T_{e}}\right) \frac{\rho_{3} \rho_{6} \times \mathcal{N}^{2}}{\mathcal{M}_{3} \mathcal{M}_{6}}\left(\mathrm{gcm}^{-3} \mathrm{~s}^{-1}\right)
\end{gathered}
$$

The electronic impact ionization kinetic constants in the forward and backward cases, are respectively :

$$
\begin{gathered}
\left(K_{e-i}\right)_{i}=2.2 \times 10^{-10} \times T_{e}^{0.5}\left(\frac{I_{H}}{E^{+}}\right)^{2} \frac{\rho_{i} \rho_{6} \times \mathcal{N}^{2}}{\mathcal{M}_{i} \mathcal{M}_{6}} \exp \left(-\frac{E^{+}}{k T_{e}}\right)\left(\mathrm{gcm}^{-3} \mathrm{~s}^{-1}\right) \\
\left(K_{i-e}\right)_{i}=10^{-25} \times T_{e}^{-1}\left(\frac{I_{H}}{E^{+}}\right)^{2}\left(\frac{\rho_{i} \times \mathcal{N}}{\mathcal{M}_{6}}\right)^{3}\left(\mathrm{gcm}^{-3} \mathrm{~s}^{-1}\right)
\end{gathered}
$$

The kinetic constant correponding to the photoionization process is:

$$
K_{p h}=7.9 \times 10^{-18}\left(\frac{I_{H}}{E^{+}}\right)^{0.5}\left(\frac{E^{+}}{h \nu}\right)^{3}\left(\frac{\rho_{3} \times \mathcal{N}}{\mathcal{M}_{5}}\right) I(x, t)\left(g_{c m}^{-3} s^{-1}\right)
$$

where $I(x, t)$ is the laser intensity at position $x$ and time $t$. The transmission and absorption of the laser by the ablated matter cloud is described below.

We define $E_{e c h}, E_{I B}, E_{\text {ioni }}, E_{p h}$, and $E_{i m p}$ to be the energy exchange rate between electrons and heavy particles, the energy absorbed by inverse Bremsshtrahlung, the ionization energy by electronic impact, the 
photoionization of the excited state energy, and the excitation by electronic impact energy, respectively, where

$$
\begin{gathered}
E_{e c h}=\frac{3}{2}\left(\frac{\rho_{6} \times \mathcal{N}}{\mathcal{M}_{6}}\right) k \frac{T_{e}-T}{\tau}, \\
E_{I B}=\alpha(x, t) \times I_{a b s}(x, t), \\
\left(E_{\text {ioni }}\right)_{i}=\left(\frac{2}{3}\left(K_{i-e}\right)_{i} e^{r e c}-\left(K_{i-e}\right)_{i} E^{+}\right)\left(\frac{\mathcal{N}}{\mathcal{M}_{i}}\right) \\
E_{p h}=K_{p h}\left(h \nu-E^{+}\right) \frac{\mathcal{N}}{\mathcal{M}_{3}}
\end{gathered}
$$

and

$$
E_{i m p}=\left(K_{e-f}-K_{f-e}\right) E^{*} \frac{\mathcal{N}}{\mathcal{M}_{3}}
$$

where $\tau$ is the electronic relaxation time,

$$
\tau=\frac{7 \times 10^{3} \times T_{e}^{1.5}}{\ln \Lambda\left(\frac{\rho_{6} \mathcal{N}}{\mathcal{M}_{6}}\right)}
$$

$\Lambda$, is the number of electrons in the Debye sphere,

$$
\Lambda=\frac{3 \times\left(k T_{e}\right)^{1.5}}{2 \times(4 \pi)^{0.5} e^{3}\left(\frac{\rho_{6} \mathcal{N}}{\mathcal{M}_{6}}\right)^{0.5}} ;
$$

The laser intensity transmission through the ablated cloud follows the Beer-Lambert law,

$$
I(x, t)=I_{0}(t) e^{-\alpha x},
$$

where $I_{0}$ is the incident laser intensity. At high temperature a particle can emit a photon, and so the absorption coefficient must be multiplied by a correction factor introduced by Rosen et al, ${ }^{17}$

$$
C_{E S}=1-\exp \left(-\frac{h \nu}{k T_{e}}\right) \text {. }
$$

Incorporating the stimulated emission coefficient, $C_{E S}$, the laser absorbed intensity becomes

$$
I_{a b s}(x, t)=I_{a b s}(x, t) C_{E S} .
$$

$\alpha$ is the Inverse Bremsstrahlung absorption coefficient, which incorporates electron-neutral and electronion absorptions; $I_{a b s}$ is the absorbed intensity by one cell in the gaseous domain.

The Inverse Bremsstrahlung absorption coefficient has two components: electron-neutral and electron-ion. One difficult problem with Inverse Bremsstrahlung absorption coefficients is the wide discrepancy between the formulations found in literature. For our calculations, and assuming the plasma to be uniform, we used the following coefficients:

- Inverse Bremsstrahlung coefficient for ions: ${ }^{13}$

$$
\alpha_{e-i}\left[C u^{+Z}\right]=(Z)^{2} 6.1 \times 10^{-29} \frac{\lambda^{3}}{\sqrt{k T_{e}}}\left(\frac{\rho_{4 / 5} \rho_{6}}{\mathcal{M}_{4 / 5} \mathcal{M}_{6}}\right) \times \mathcal{N}^{2}\left(\mathrm{~cm}^{-1}\right),
$$

where $Z$ is the charge of the species, i.e., 1 for $\mathrm{Cu}^{+}$and 2 for $\mathrm{Cu}^{+2}$.

- Inverse Bremsstrahlung coefficient for neutrals: ${ }^{15}$

$$
\alpha_{n-e}=6.1 \times 10^{-29} \frac{\lambda^{3}}{\sqrt{T_{e}}}\left(\frac{\rho_{6} \mathcal{N}}{\mathcal{M}_{6}}\right)\left(\frac{\rho_{1} \mathcal{N}}{\mathcal{M}_{1}}\right) \quad\left[\frac{\pi}{15 \sqrt{3}}\right]\left[\frac{2 . I_{H}}{h \nu+T_{e}}\right]\left(\mathrm{cm}^{-1}\right)
$$


The electron-ion coefficient, is dominant once the plasma is created. However, the electron-neutral absorption coefficient must also be considered to correctly describe plasma breakdown.

Hence, the total inverse Bremsstrahlung coefficient is

$$
\alpha=\alpha_{e-n}+\alpha_{e-i}\left[C u^{+}\right]+\alpha_{e-i}\left[C u^{+2}\right]
$$

The source terms of the mass conservation equation corresponding to each species are giving as follows :

$$
\omega_{i}\left\{\begin{array}{c}
\omega_{1}=K_{e-f}-K_{f-e} \\
\omega_{2}=0 \\
\omega_{3}=K_{f-e}-K_{e-f}-K_{p h}-\left(K_{e-i}\right)_{3}+\left(K_{i-e}\right)_{3} \\
\omega_{4}=-\left(K_{i-e}\right)_{4}+\left(K_{e-i}\right)_{4}+\left(K_{e-i}\right)_{3}-\left(K_{i-e}\right)_{3} \\
\omega_{6}=K_{p h}+\left(K_{e-i}\right)_{3}-\left(K_{i-e}\right)_{3}+\left(K_{e-i}\right)_{4}-\left(K_{i-e}\right)_{4}
\end{array},\right.
$$

The source terms of the heavy particles and electronic conservation energy are given as follows:

$$
\omega_{E} / \omega_{E_{e}}\left\{\begin{array}{c}
\omega_{E}=E_{e c h} \\
\omega_{E_{e}}=E_{I B}-E_{\text {ech }}+E_{\text {ioni }}+E_{p h}+E_{i m p}
\end{array} .\right.
$$

\section{Boundary conditions and thermal and hydrodynamical model coupling}

The space-time history simulated in this study is sufficient to assume that the outer boundary of the simulated space-time profile is not significantly affected by the end of the laser pulse. Thus the boundary conditions at the origin of the simulated history are simply the initial conditions, as given by ${ }^{12}$

$$
\begin{gathered}
T\left(x_{\text {end }}\right)=T_{0}=T(t=0 s)=300 K . \\
T_{e}\left(x_{\text {end }}\right)=T e_{0}=T_{e}(t=0 s)=300 K .
\end{gathered}
$$

For the surface that receives the laser flux, the boundary condition is zero flux for the solid or liquid phase, because there are no particle or energy fluxes until boiling begins,

$$
\begin{aligned}
& \left(\frac{\partial T}{\partial x}\right)_{x=0}=0 . \\
& \left(\frac{\partial T_{e}}{\partial x}\right)_{x=0}=0 .
\end{aligned}
$$

The coupling of the target and the plume is insured through the boiling temperature, the plasma shielding effect and the mass flow of the vaporized matter.

- The boiling temperature depends on the pressure of the plume at the material surface. This dependency is expressed by the Clausius-Clapeyron equation:

$$
T_{e b}=\frac{1}{\left(\frac{1}{T_{0}}-R \frac{\ln \frac{P_{S}}{P_{0}}}{\Delta H}\right)}
$$

$T_{0}$ : is the boiling temperature, $P_{0}$ : the atmospheric pressure, $P_{s}$ : the plume pressure, $\Delta H$ : the latent heat of vaporization and $R$ the ideal gas constant.

- The plasma shielding effect which is modeled by equation (14) and (20). The amount of energy that can actually reach the target surface depends on how much of the laser beam is absorbed by the plume. 
- The mass flow of the laser vaporized material. Before the start of the evaporation process, the adiabatic conditions $(30,31)$ is used at the interface between the target and the background environment. Once the evaporated matter leaves the surface material, the mass flow of the vaporized matter is calculated, it is given by:

$$
\dot{m}=\rho_{l i q}\left(\frac{d x}{d t}\right)_{\text {evap }}
$$

where $\left(\frac{d x}{d t}\right)_{\text {evap }}$ is the variation over time of the thermal ablation depth.

The mass flow of the electrons contained in the evaporated matter from the target is calculated is given by:

$$
\dot{m}_{e}=\rho_{e(l i q)}\left(\frac{d x}{d t}\right)_{\text {evap }}
$$

A total mass, momentum and energy balance is done at the first cell of the gaseous domain. The behavior of the evaporated matter is modeled afterwards by the Euler equations.

The space-time computational domain is considered large enough to assume its outer boundary unaffected by the laser pulse absorption and heat diffusion. This commonly admitted boundary condition is expressed as follow ${ }^{5,8-10}$

\section{Numerical approach}

To solve the thermal model, we chose an explicit central difference scheme, second order precise in space and first order precise in time. ${ }^{5}$ The time step was to $\Delta t=10^{-14} \mathrm{~s}$, and the space step was $\Delta x=10^{-6} \mathrm{~cm}$.

The hydrodynamic aspect of our modeling approach was solved using the LCPFCT algorithm. ${ }^{18,19}$ This algorithm is based upon a finite difference scheme associated with general methods for flux corrected transport, and is a predictor corrector numerical scheme accurate to the fourth order in space and second order in time. ${ }^{5}$ A uniform mesh was used with space step $\Delta x=10^{-4} \mathrm{~cm}$ and time step $\Delta t=10^{-14} \mathrm{~s}$.

\section{Results}

In order to compare our models to experimental datas and previous modeling some plasma ignition threshold have been determined. The results are sum up in table 1. We can clearly see that our new model have a better agreement with measurements than the previous one, that shows that even for short pulsed interaction with metals electronic non equilibrium plays a key role. Calculations have been carried out under low pressure $(10 \mathrm{~Pa})$ to simulate the vacuum condition of the experiments.

\begin{tabular}{|l|l|l|l|}
\hline Laser beam & Experimental threshold & Numerical(old model) & Numerical(new model) \\
\hline $193 \mathrm{~nm}, 8 \mathrm{~ns}$ & $1 \mathrm{~J} / \mathrm{cm}^{2}$ & $0.84 \mathrm{~J} / \mathrm{cm}^{2}(16 \%)$ & $1.1 \mathrm{~J} / \mathrm{cm}^{2}(10 \%)$ \\
\hline
\end{tabular}

Table 1. Plasma ignition threshold comparison with experiments and old model

One of the main relevant outputs when dealing with laser ablation is the ablated depth. In figure one we have a striking result : there is almost a factor 2 between the ablated depth results obtained by our old model and the new one taking into account the electronic non equilibrium. With the new models the ablated depth is much smaller. We need to have a closer look at other results to try to validate and understand this quite big difference.

Figure 2 shows us that the shielding is much important with the old model, that quite logical as int this cas we have almost twice more ablated mass and so the plume is able to absorb much more energy from the laser. This assumption is sustained by the Figure 3 in which we can see that the plasma characteristics are quite similar at the very beginning of the plasma evolution, after that differences appeared that are due to the difference in the plasma shielding time evolution. 


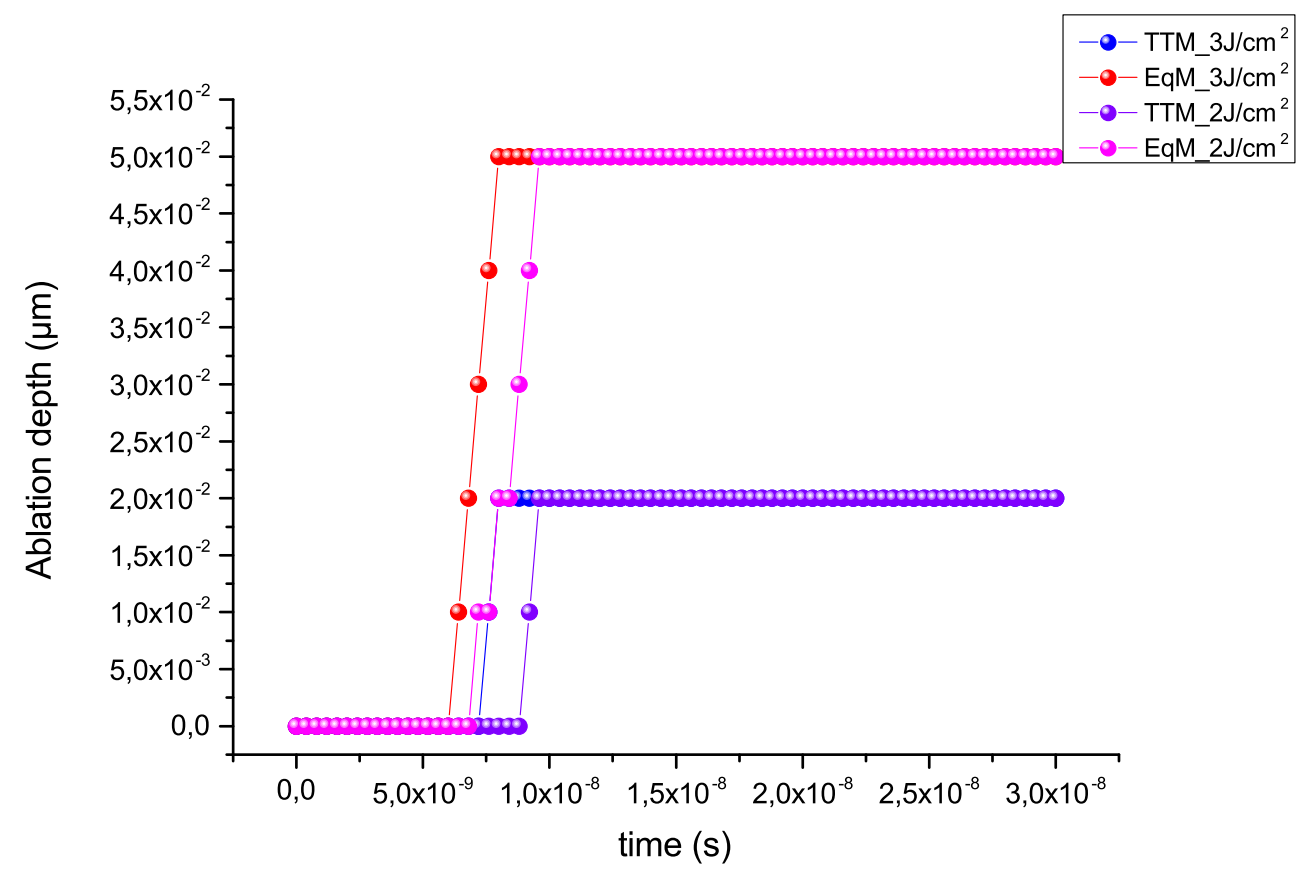

Figure 1. Ablation depths predictions comparison between the two temperature thermal model and the equilibrium thermal model.



Figure 2. Plasma shielding comparison between equilibrium and out of equilibrium plume initialization for two beam fluences 


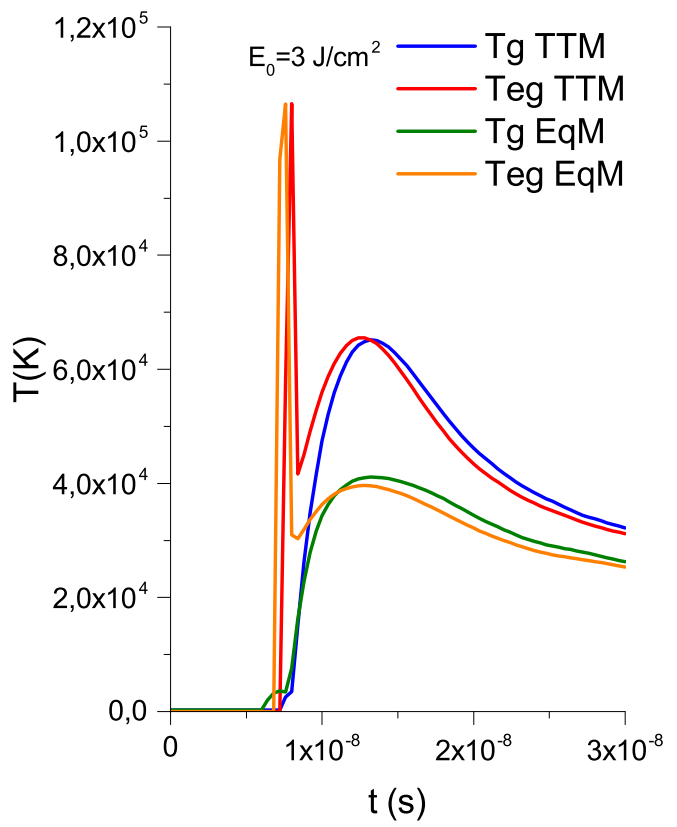

Figure 3. Electron and heavy species temperature near the target surface

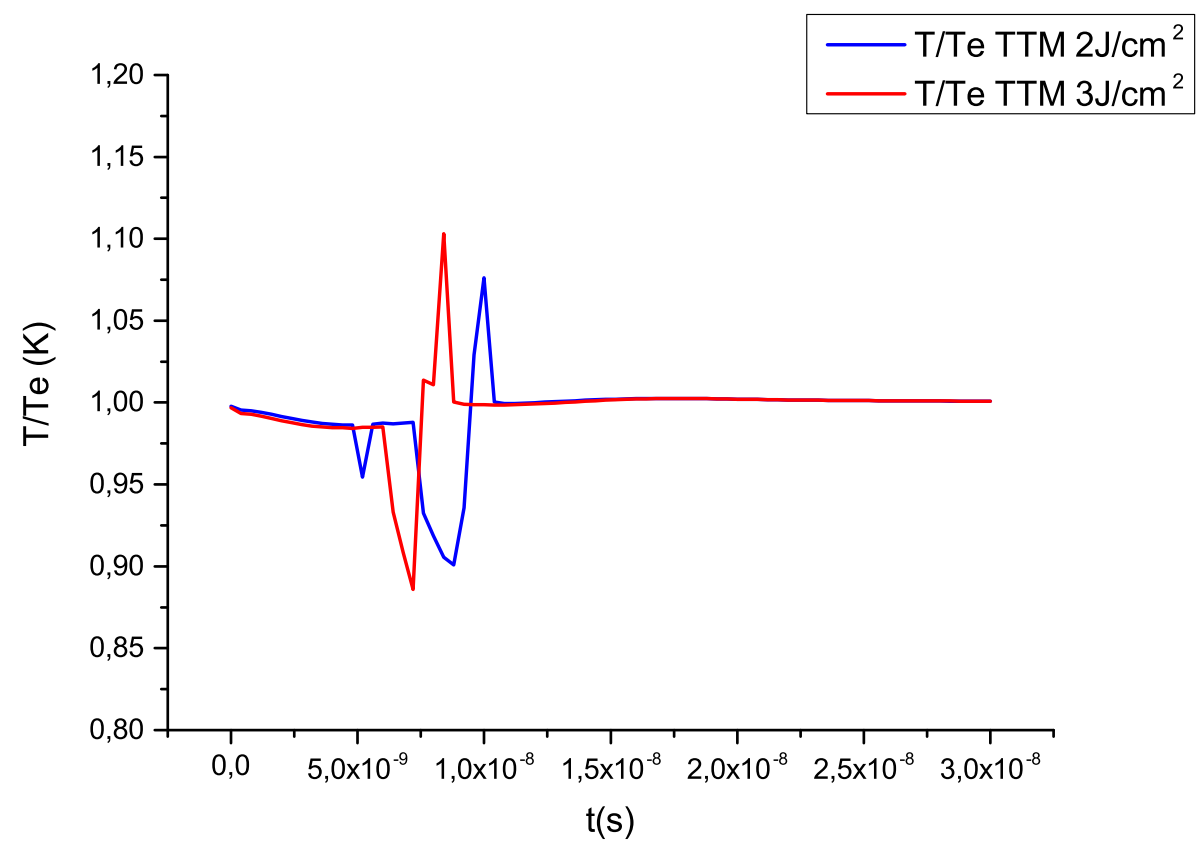

Figure 4. Non-equilibrium thermal ratio for two laser beam fluences 


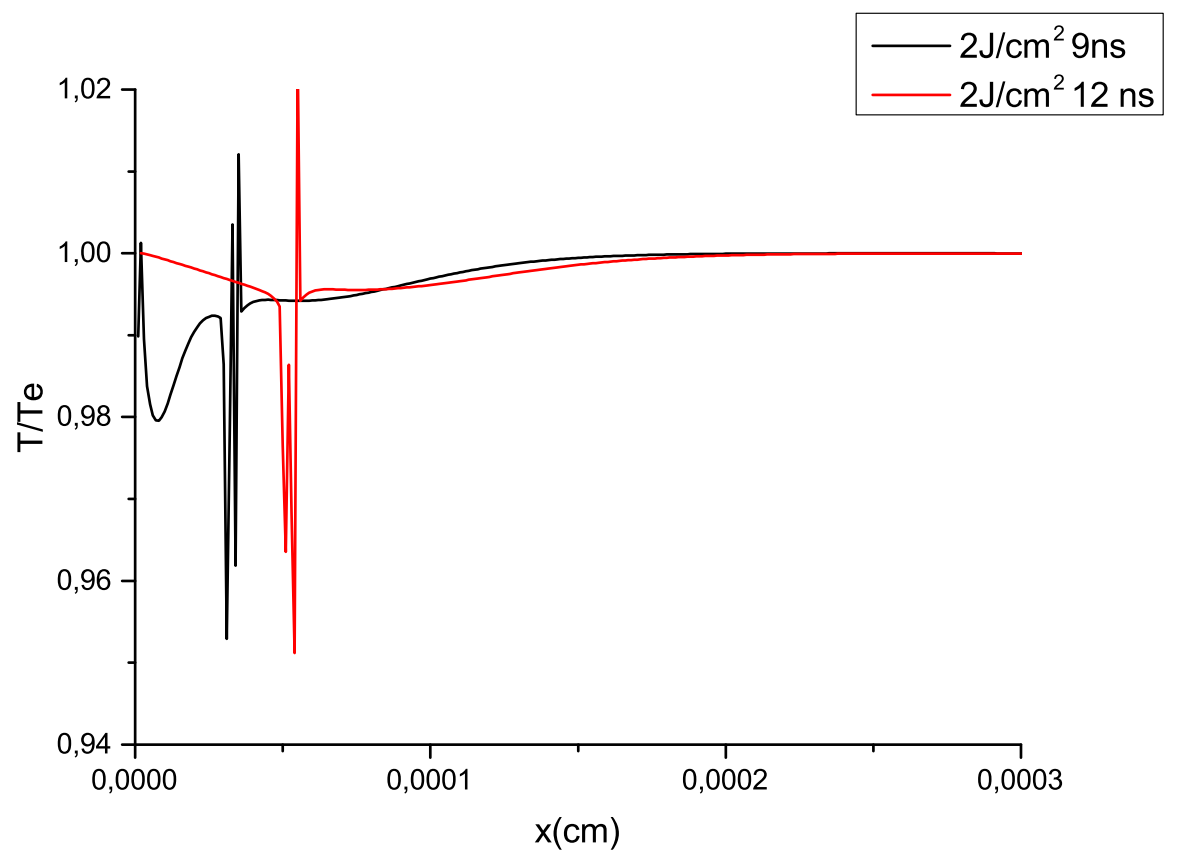

Figure 5. Non-equilibrium thermal ratio inside the target material for a $2 \mathrm{~J} / \mathrm{cm}^{2}$ laser beam fluence at 9 and $12 \mathrm{~ns}$

In order to go further in our investigations we are going to have a look of what occurs in the target. In Figure 4 the electronic non equilibrium is clearly seen and we could also observed its evolution. At the the beginning the electron are excited by the laser pulse and then exchange energy with the lattice in order to heat the target, this could explain the differences with the two models because a part of the laser energy is not directly used in the target heating and a part of it is in somehow wasted. In this figure we could also see the diffusion of the energy and of the electronic non equilibrium into the depth the material that phenomena could also explain the lower ablated depth in the electronic non equilibrium case. It also notable that the overall affected depth by electronic non equilibrium is for the two times roughly 2 microns.

Lets now move to the characteristics and dynamic of the ablated materials. In figure 6 one can notice that the two plumes have more or less the same same characteristics excepted for their dynamics : velocity, expansion distance and shock wave propagation. It is clearly demonstrated in figure 6 and is easily explained by the fact that in the old model there is more ablated mass and reinforced by the much bigger plasma shielding in this case, so we ended with a plasma in which we injected much more energy and so its expansion is much more violent. One very intresting thing is that this energy is converted is kinetic energy and not in internal energy (temperature or ionization). This feature is confirmed in figure 7 and 8 and also confirmed that the shock wave is much stronger in the old model case. Another interesting thing is that the ionization degree is a little bit higher in our new models but it is more than logical as in this model the ablated material are injected in the plasma with already an electronic temperature non equal to the heavy particle temperature so it will enhance the ionization process.

Lets have a closer look to the different species density in figure 9. These results can be directly related to the temperatures (heavy and electronic) as the curves follow the same pattern.It is not such a surprise but it clearly means that the photinozation process do not play a key role after the plasma breakdown and during the laser-plasma interaction.

In figure 10 the shock wave propagation is observed and it is clearly confirmed that the shock wave generated with the old model is the strongest one. 


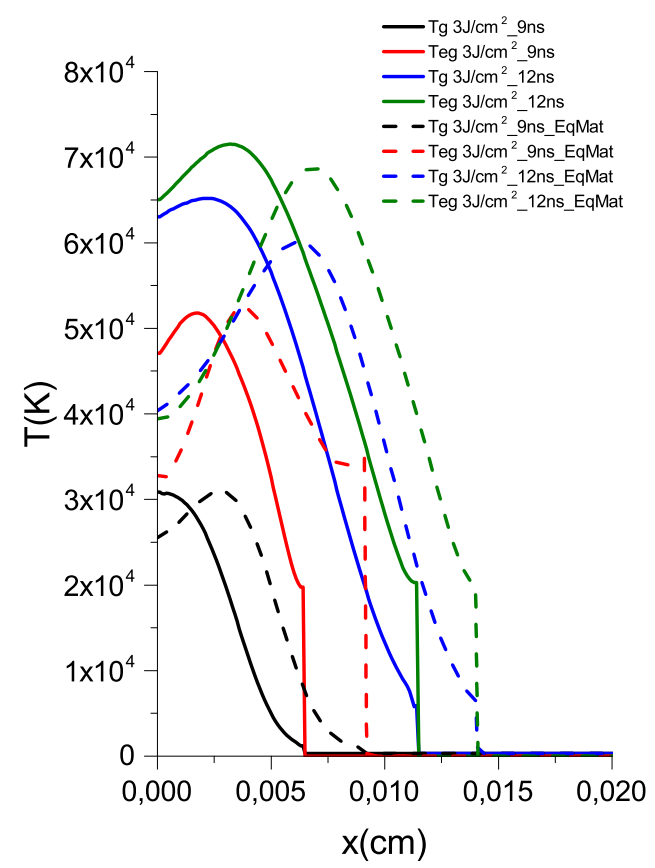

Figure 6. Electronic and heavy species temperatures comparison between equilibrium and out of equilibrium plume initialization for two beam fluences

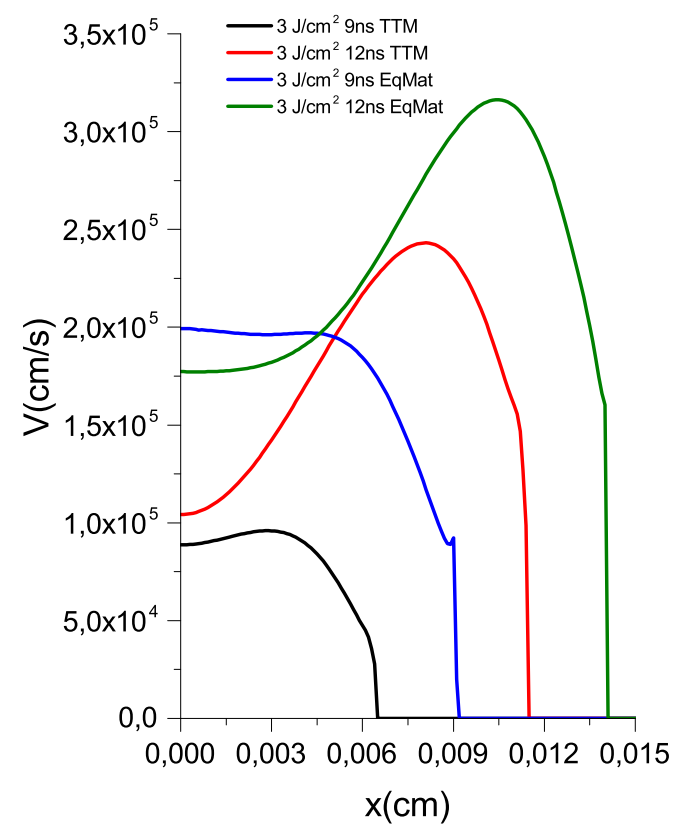

Figure 7. Plume expansion velocity comparison between equilibrium and out of equilibrium plume initialization for two laser beam fluences. 


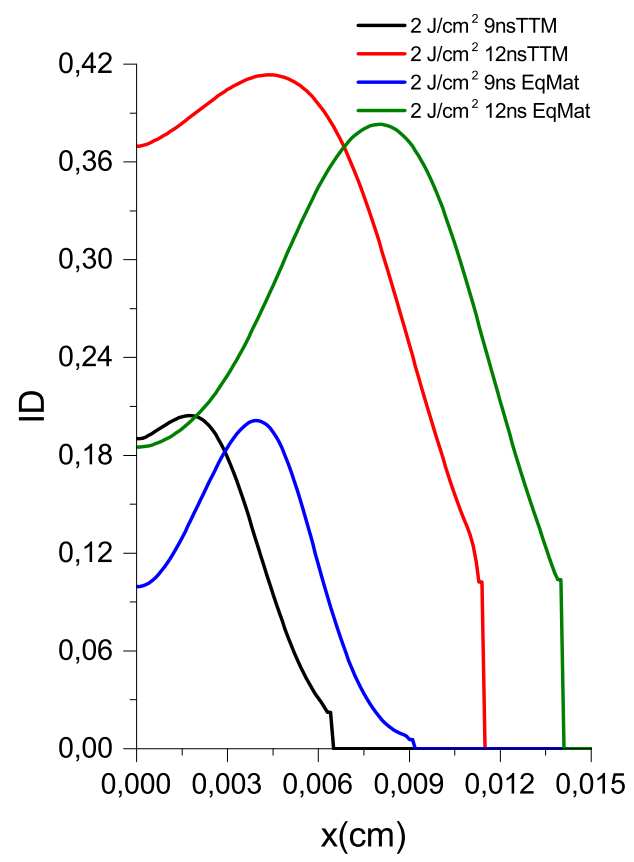

Figure 8. Ionization degree comparison between equilibrium and out of equilibrium plume initialization for two laser beam fluences.

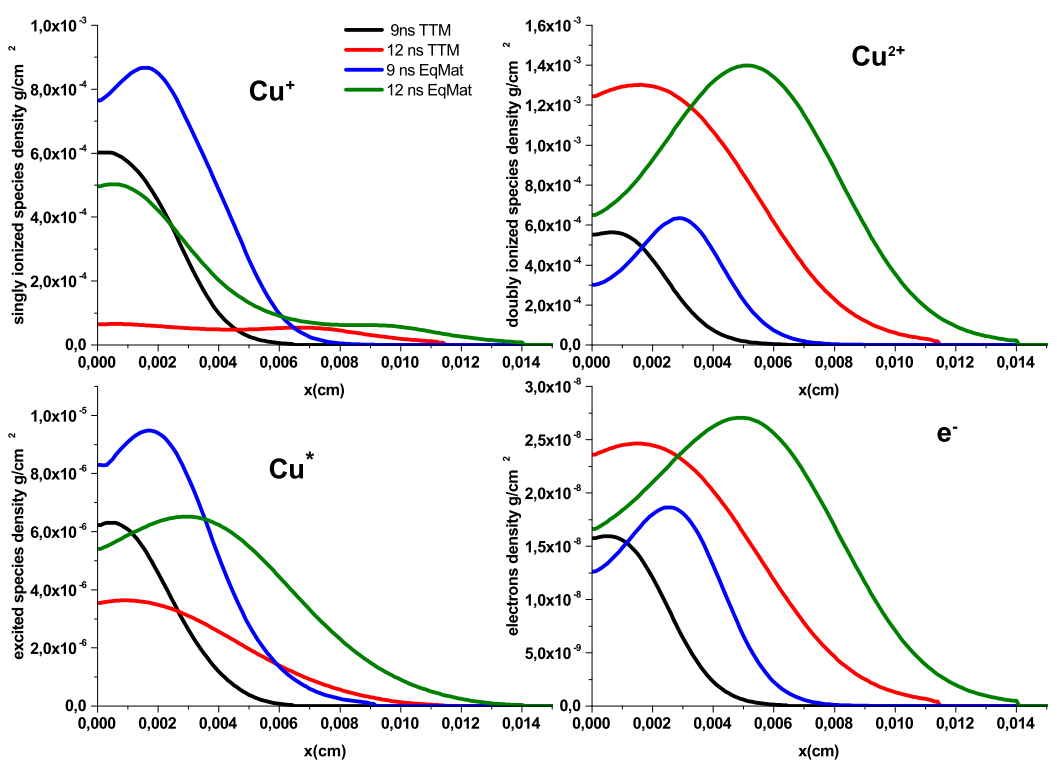

Figure 9. Species variations comparison between equilibrium and out of equilibrium plume initialization for a laser beam fluence of $3 \mathrm{~J} / \mathrm{cm}^{2}$ 


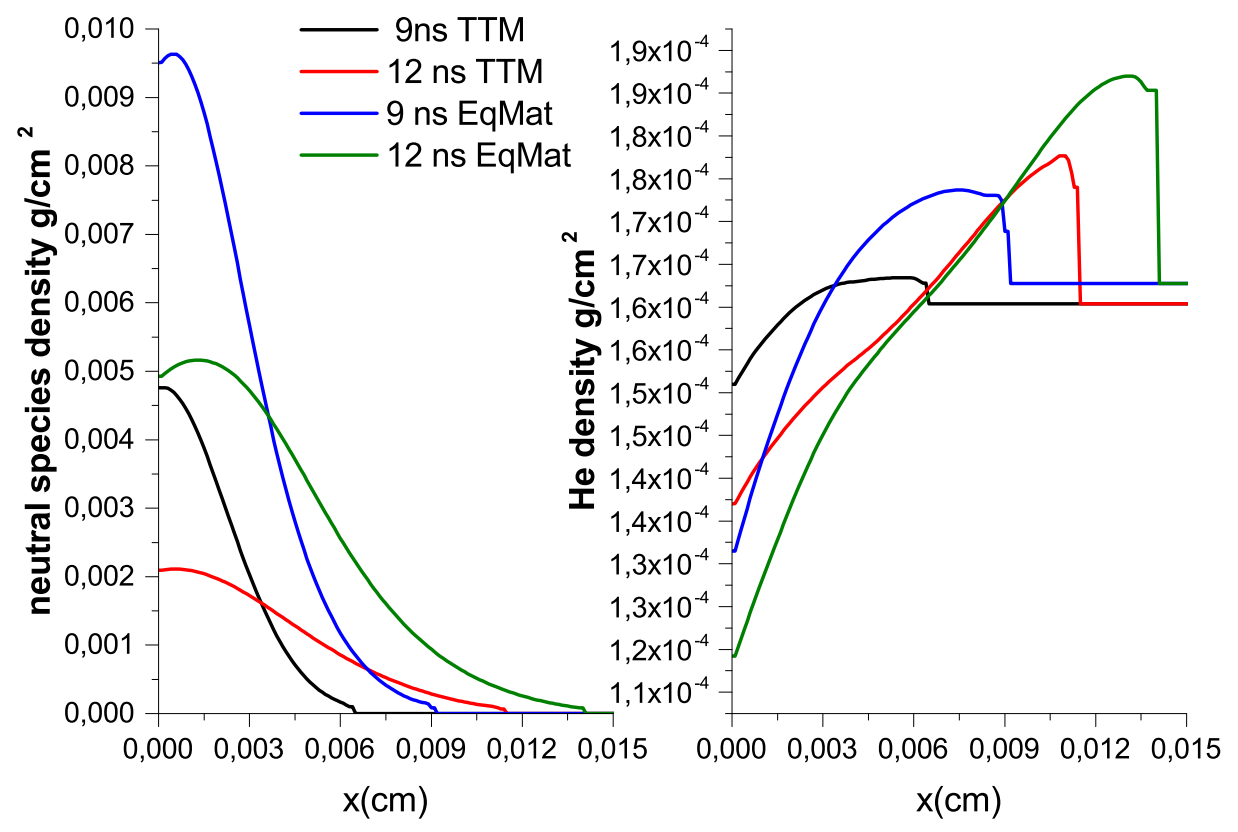

Figure 10. Neutral and background gas species variations comparison between equilibrium and out of equilibrium plume initialization for a laser beam fluence of $3 \mathrm{~J} / \mathrm{cm}^{2}$

\section{Conclusion and perspectives}

An electronic non equilibrium model for a metal material for laser-matter interaction has been developed and implemented in our in house numerical code. The first results are very promising and the main features is that this new model has a big impact on the ablated depth in order to definitely validate this result and in order to have a deeper and clearer understanding we need to do more calculations and investigations and also to try to find more experimental data

\section{References}

\footnotetext{
${ }^{1}$ Stefan Scharring, Lukas Eisert, Raoul-Amadeus Lorbeer, and Hans-Albert Eckel Momentum predictability and heat accumulation in laser-based space debris removal Optical Engineering 58(1), 011004 (January 2019)

${ }^{2}$ Stefan Scharring, Jascha Wilken, and Hans-Albert Eckel Laser-based removal of irregularly shaped space debris Optical Engineering 56(1), 011007 (January 2017)

${ }^{3}$ Raoul-Amadeus Lorbeer, Michael Zwilich, Miroslav Zabic, Stefan Scharring, Lukas Eisert, Jascha Wilken, Dennis Schumacher, Markus Roth and Hans-Albert Eckel Experimental verification of high energy laser-generated impulse for remote laser control of space debris SCIENTIFIC REPOSRTS (2018) 8:8453

${ }^{4}$ Bruno Esmille, Christophe Jacque lard, Hans-Albert Ecke, and Edwin Wnuk Space debris removal by ground based laser Main conclusions of the European project CLEANSPACE HPLA/BEP 2014

${ }^{5}$ J.D. Parisse M. Sentis D.E.Zeitoun, Modeling and numerical simulation of laser matter interaction and ablation with 193 nanometer laser for nanosecond pulse, International Journal of Numerical Methods for Heat and Fluid Flow,21:73 - 94, 2011.

${ }^{6}$ Carsten Shäfer and Herbert M. Urbassek, Metal ablation by picosecond laser pulses: A hybrid simulation, PHYSICAL REVIEW B 66, 115404-2002

${ }^{7}$ A.K. Ait oumeziane, A.Sari, B.Liani, J.D.Parisse. Theoretical and numerical study of the interaction of a nanosecond laser pulse with a copper target for laser-induced breakdown spectroscopy applications Journal of the Optical Society of America B, Vol. 31, No. 1, 2014

${ }^{8}$ A.K. Ait oumeziane, B.Liani, J.D.Parisse. Laser induced plasma on copper target, a non-equilibrium model Physics of Plasmas 21, $023507(2014)$

${ }^{9}$ A.K. Ait oumeziane, B.Liani, J.D.Parisse. Laser-Induced Plasma on a Titanium Target, a Non-equilibrium Model Plasma Chemistry and Plasma Processing, 36(2), 711-730.

${ }^{10}$ A.K. Ait oumeziane, B.Liani, J.D.Parisse. Non-equilibrium modeling of UV laser induced plasma on a copper target in the presence of $\mathrm{Cu}^{2+}$ Physics of Plasmas, 23(3), 033502 .
} 
${ }^{11}$ A.K. Ait oumeziane, J.D.Parisse. Toward a comprehensive UV laser ablation modeling of multicomponent materials - a non-equilibrium investigation on titanium carbide Physics of Plasmas 25, 053511 (2018)

${ }^{12} \mathrm{H}$. Van Driel Kinetics of high density plasmas generated in Si by 1.06 and 0.53 micrometer picosecond laser pulses, Physical review B, 35, 81661986.

${ }^{13}$ A.L. Thomann, C. Boulmer-Leborgne and B. Dubreuil A contribution to the understanding of the plasma ignition mechanism above a metal target under UV laser irradiation, Plasma Sources Sci. Technol. 6 298-306 1997.

${ }^{14}$ C.L.Liu J.N.Leboeuf, R.F.Wood D.B.Geohegan J.M.Donato K.R.Chen A.A.Puretzky Vapor breakdown durinng ablation by nanosecond laser pulses, Materials research society Symposium Proceding, 388, 133.

${ }^{15}$ Ya. B. Zel'dovitch and Yu. P. Raizer Physics of Shock Waves and High Temperature Hydrodynamics Phenomena, Academic Press, New York 1966.

${ }^{16}$ D.I. Rosen, J. Mitteldorf, G. Kothandaraman A.N. Pirri and E.R. Pugh Coupling of pulsed 0.35 micrometer laser radiation to aluminum alloys, J. Appl. Phys., 53 (4), 31901982.

${ }^{17}$ D.I Rosen, D.E. Hastings and G.M. Weyl, J. Appl. Phys. 53, 58821987.

${ }^{18}$ E.S. Oran and J.P. Boris Numerical Simulation of Reactive Flow Elsevier, New York, 1987.

${ }^{19}$ J.P. Boris, A.M.Landsberg, E.S. Oran and J.H. Garner LCPFCT a flux corrected transport algorithm for solving generalized continuity equations Laboratory for computational physics and fluid dynamics, Naval Research Laboratory, Report Avril 1993. 